\title{
Article
}

\section{Effects of physical efforts on injury in elite soccer}

Carling, C., Le Gall, F., and Reilly, T.P.

Available at http://clok.uclan.ac.uk/5569/

Carling, C. ORCID: 0000-0002-7456-3493, Le Gall, F., and Reilly, T.P. (2010) Effects of physical efforts on injury in elite soccer. International Journal of Sports Medicine, 31 (03). pp. 180-185. ISSN 0172-4622

It is advisable to refer to the publisher's version if you intend to cite from the work. http://dx.doi.org/10.1055/s-0029-1241212

For more information about UCLan's research in this area go to http://www.uclan.ac.uk/researchgroups/ and search for < name of research Group>.

For information about Research generally at UCLan please go to http://www.uclan.ac.uk/research/

All outputs in CLoK are protected by Intellectual Property Rights law, including Copyright law. Copyright, IPR and Moral Rights for the works on this site are retained by the individual authors and/or other copyright owners. Terms and conditions for use of this material are defined in the policies page.

\section{CLoK}

Central Lancashire online Knowledge www.clok.uclan.ac.uk 
This is a pre-proof corrected manuscript, as accepted for publication, of an article

December 2009, available online:

4

https://www.thieme-connect.com/DOI/DOI?10.1055/s-0029-1241212

PLEASE REFER TO THE PUBLISHED VERSION FOR CITING PURPOSES

6

7

10

11

12

13

14

15

16

17

18

19

20

21 Corresponding Author: Christopher Carling, Ecole des Metiers du Sport Professionnel,

22 Domain de Luchin, Camphin-en-Pévèle, 59780, France. Tel: +33 14891 07 93. E-mail:

23 chris.carling@free.fr

24

25

\section{Effects of physical efforts on injury in elite soccer}

Christopher Carling ${ }^{1}$, Franck le Gall ${ }^{2}$, and Thomas Reilly ${ }^{3}$.

${ }^{1}$ Ecole des Metiers du Sport Professionnel, LOSC Lille Métropole Football Club, Domain de Luchin, Camphin-en-Pévèle, 59780, France,

${ }^{2}$ LOSC Lille Métropole Football Club, Domain de Luchin, Camphin-en-Pévèle, 59780, France,

${ }^{3}$ Research Institute for Sport and Exercise Sciences, Liverpool John Moores University, Henry Cotton Campus, 15-21 Webster Street, Liverpool L3 2ET, UK.

Running head: Physical performance and soccer injury 


\section{$1 \quad$ Abstract}

2 In this study, the influence of physical efforts on occurrence of match injury in a 3 professional soccer club was investigated. Computerised motion-analysis was used to 4 measure the physical efforts of players during 10 injury situations. Total distance and 5 those covered at different movement intensities were measured across the 5-minute 6 period preceding injury. If the final run preceding injury involved a high-intensity 7 action (HIA), the distance, duration and speed of the effort and the recovery time 8 between this and the penultimate HIA were measured. To determine the influence of 9 these physical efforts, the results were compared to a normative profile for players 10 computed from data across 5 games for the same variables; habitual distances covered 11 over a 5-minute period and characteristics of and recovery time between HIA. 12 Compared to the normative profile, no differences were reported in physical 13 characteristics during the period leading up to injury or for HIA although the latter were 14 substantially higher in intensity (duration and distance). A lower than normal recovery 15 time between HIA prior to injury was observed $(35.6 \pm 16.8 \mathrm{~s}$ versus $98.8 \pm 17.5 \mathrm{~s}$, $16 \mathrm{p}=0.003)$. Within the limitations of the small sample, these findings may aid in further 17 understanding injury and physical performance in elite soccer.

21 Key terms: injuries, motion analysis, football incident analysis, fatigue 


\section{Introduction}

2 Soccer (Association Football) is a complex contact sport with high physical, technical,

3 tactical, and physiological demands at the elite level [10], and the risk of injury is

4 considerable [26]. In order to suggest preventive strategies specific to soccer, it is

5 necessary to have detailed information on the injury mechanisms involved. Previously,

6 analysis of soccer incidents that combines game-specific and medical information has

7 been employed to describe how injuries and high-risk situations of injury occur in

8 match-play [1,2,3,5,23]. Examination of the playing situation, athlete-opponent

9 interaction, and refereeing has highlighted the importance of tackling duels and heading

10 duels as high risk situations. In addition, a critical incident technique was designed to

11 identify behaviour with potential injury risk [32]; the findings emphasised tackling,

12 receiving a tackle and charging an opponent as the circumstances with the highest injury

13 potential.

14 A precise description of the inciting event is a key component in understanding

15 the causes of injuries in sport [7]. In soccer, there is a need to investigate the effects of

16 the physical efforts of players on the occurrence of injury [9]. The physical demands of

17 match-play can now be easily and accurately investigated via information collected

18 using computerised motion-analyses of player movements. Yet, to date, the effects of

19 movement intensity when injuries are incurred has received scant attention.

20 Furthermore, in the studies that have touched on this area $[1,2,3,5]$, movement

21 intensities were recorded subjectively and no comprehensive data were provided on

22 specific running characteristics such as starting, average or maximal running speed or

23 on the length or duration of actions. Similarly, no study has examined the possible

24 effects of physical efforts prior to the injury situation. Related research by Rahnama et

25 al. [32] reported an increase in critical incidents and injury risk in the first $15 \mathrm{~min}$ and in 
1 the last 15 min compared to other periods of the game. The presumption was that injury

2 risk was associated to the periods in which exercise intensity was highest when players

3 were fresh and lowest when players experience fatigue during the game, but there were

4 no measurements of players' movements recorded. As players commonly experience

5 transient fatigue in match-play notably after periods of intense exercise [8,28], motion

6 analysis techniques could be used to determine whether greater than usual periods of

7 intense exercise or inadequate recovery time between high-intensity efforts predispose a

8 player to injury $[9,10]$.

9 Therefore, the aim of this investigation in an elite soccer club was to examine

10 the physical efforts of players in the period leading up to and for the final running action

11 in occurrences of injury in professional match-play.

13 Methods

14 In the present study, injuries sustained during competitive matches (a total of 54

15 League matches from mid-season 2007-08 to end of season 2008-09) in players

16 belonging to a 1st Division French League Club had been prospectively diagnosed and

17 documented by the team's physician in a sports injury database (TeamSanté, Enora

18 Technologies, Paris, France). Player consent and local ethics committee approval were

19 obtained.

The methodologies and definitions of injury used in the present study closely

21 follow those recommended by International Soccer Injury Consensus Groups [16,22]. A

22 total of 47 injuries forcing a layoff of over 48 hours not including the day of injury

$23[24,29]$ were available for analysis. Information including the type, location and cause

24 of injury and the match period during which each injury occurred (matches were

25 divided into six 15-minute periods, i.e., 0-15min, 15-30mins and 30-45mins for each 
1 respective half) was available. Also stored in the database was information on whether

2 the player was forced to leave the field due to injury and the total number of days the

3 player could not participate fully in training and competition. This latter number was

4 used to calculate the severity of injury [16]. The severity of each injury was defined as

5 slight, minor, moderate, or major depending on whether the player was absent from

6 training or competition for two to three days, four to seven days, one to four weeks, or

7 more than four weeks respectively [24,29]. Finally, descriptive information on the final

8 action at the time the injury was sustained and whether contact had occurred between

9 players was recorded.

10 The occurrence of injuries in soccer match-play cannot always be clearly 11 identified on video recordings [27]. Often there is no stoppage in play and players do 12 not go down on the pitch to receive treatment and are subsequently substituted at half13 time or receive treatment after the match [1]. Therefore, only injuries that had forced the 14 player to immediately leave and not return to the field of play were included for 15 investigation. This process also helped avoid the inclusion of situations where players 16 intentionally lay down either to rest, feign injury or delay playing time [17]. To reduce 17 the effects of opposition behaviour, only injury incidents where no foul-play was observed (decision by referee to award a free-kick) were considered. These strict inclusion criteria limited the total number of injuries to 17 (36.2\% of the total injury 20 sample obtained over the two seasons).

After retrospective review of the medical records by the same club physician

22 who had diagnosed the injuries, each injury incident was cross-referenced to determine whether the match had been recorded and analysed by the multiple-camera player tracking system (AMISCO Pro, Sport-Universal Process, Nice, France) used by the club to evaluate physical, technical and tactical performance in competition. A validation of 
1 this system has demonstrated high-levels of validity, accuracy and reliability in

2 measuring player movements in elite soccer play [41]. If information from the system

3 was available, the physical performance data were then used to measure the efforts of

4 players over 5-minutes leading up to and during the injury situation. To obtain the time

5 of injury, the club physician and the injured player reviewed the digital video recording

6 of the injury incident and the time corresponding to the incident was obtained from the

7 digital time code. Altogether, data were available for a total of 10 of the injuries $(21.3 \%$

8 of the total injury sample).

To investigate the effects of physical performance prior to injury, the total

10 distance and distance covered in four categories of movement intensity were measured

11 over the 5-minute period preceding the time of injury: $0-11 \mathrm{~km} / \mathrm{h}$ (walking, jogging or

$12 \mathrm{WJ}$ ); $11.1-14 \mathrm{~km} / \mathrm{h}$ (low-intensity running or $\mathrm{LI}$ ); $14.1-19 \mathrm{~km} / \mathrm{h}$ (moderate-intensity

13 running or $\mathrm{MI}$ ); $>19.1 \mathrm{~km} / \mathrm{h}$ (high-intensity running or $\mathrm{HI}$ ) [11]. To determine whether

14 performance over this 5-minute period may have influenced injury, data across five

15 completed matches were used in an attempt to establish a normative physical

16 performance profile for each player [11,12]. For this profile, the total distance covered

17 in each category of movement intensity was calculated for entire games. To calculate

18 the distance covered over a 5-minute period, the total distance covered was divided by

19 the match duration (in minutes) and then multiplied by 5 . This figure was considered to

20 be the player's habitual match-play activity level over a 5-minute period. The physical

21 performance data used for the normative profile were based on information during the

22 same season in which the injury occurred. The normative profile was subsequently

23 compared to the physical efforts over the 5-minute period prior to injury.

24 The characteristics of the final running action in each injury situation were

25 investigated. The starting and maximal running speed of actions and speed at time of 
1 injury were documented as were the duration, distance covered and average speed of the 2 movement.

3 It has been suggested that players may be more at risk of incurring injury during 4 HI activities [9]. If the player reached a speed $>19.1 \mathrm{~km} / \mathrm{h}$ for a minimum of 1 second 5 duration at any time during the final running action, then the effort was considered to be 6 a high-intensity run. Therefore, those injuries that had been identified as occurring 7 during a $\mathrm{HI}$ exercise bout were investigated to determine whether the characteristics of 8 these running actions differed from the player's habitual HI profile for this form of 9 game activity. For this purpose, a normative profile was created for the speed, distance 10 and duration of $\mathrm{HI}$ actions for five 90-minute games for each player and in which the 11 player did not incur an injury. This result was compared to the same data obtained from 12 each individual injury action.

13 Finally, to determine whether recovery time may have played a part in injury,

14 the time between the penultimate $\mathrm{HI}$ effort and the HI action leading to injury was 15 determined. Again, this result was compared to a normative profile for each player 16 created from the calculation of the average recovery time between $\mathrm{HI}$ actions over five 17 full games.

18 Statistical analyses of the dataset were performed using paired t-tests to test for 19 differences in physical activity profiles prior to and during the injury situation and those 20 obtained from the normative profiling of the same player's performances. The level of 21 accepted statistical significance was set at $\mathrm{p}<0.05$. To control the Type-I error rate 22 observed in multiple measures of physical performance in competitive soccer, a pseudo23 Bonferroni's adjustment is used through dividing the alpha level by the number of 24 categories in which objective measures of physical performance are classified $25[12,35,36]$. In this case, the three normative profiles were utilised to compare 
1 performance: physical efforts over a 5-minute period, characteristics of $\mathrm{HI}$ actions and

2 recovery time between bouts of $\mathrm{HI}$ exercise. Thus, an operational alpha level of 0.017

$3(\mathrm{p}<0.05 / 3)$ was used. Effect sizes for these differences were also determined. Effect size

4 values (using Hedge's adjustment for small sample sizes) of 0.2, 0.5 and above 0.8 were

5 considered to represent small, intermediate and large differences, respectively (13).

6

\section{$7 \quad$ Results}

8 Information on the characteristics of injuries and the final running action in each injury

9 situation is reported in Table 1.

10 Out of the 10 injuries, $60 \%$ were diagnosed as sprains while strains $(20 \%)$, 11 bruising (10\%) and a combined fracture/dislocation (10\%) were also recorded. Injuries 12 to the ankle region were more common (50\%) while the upper leg (30\%) knee (20\%) 13 were also affected. Altogether, $80 \%$ of injuries were considered to be of moderate 14 severity while the remaining part (20\%) was classed as major injuries. Finally, only two 15 injuries (20\%) were sustained in the second half and a greater proportion of injuries $16(60 \%)$ involved physical contact between players.

17 The starting speed in all but one action was observed to be at low exercise intensity. Altogether, 8 out of 10 of the final running efforts before the injury was incurred involved a HI run $(>19.1 \mathrm{~km} / \mathrm{h})$ and the average speed of movement of these

20 final actions was within the moderate-intensity range $(\sim 17 \mathrm{~km} / \mathrm{h})$. In a third of the 21 movements $(33.3 \%)$, the speed at the time the injury was sustained corresponded to 22 moderate intensity.

Insert Table 1 about here. 
The total distance and the distance covered at different intensities of movement

2 in the 5-minutes prior to injury compared to the habitual physical performance profile of

3 players are presented in Table 2. The lack of significant differences reported for any of

4 these measures of physical performance were accompanied by insignificant to

5 intermediate effect size differences. However, players covered around a third more

6 distance (35\%) at high running intensities before sustaining an injury compared to

7 typical performance over a 5-minute period.

Also presented in Table 2 is information on the average speed, length, duration

9 and recovery time of the eight $\mathrm{HI}$ actions that led to injury compared to the habitual

10 characteristics of $\mathrm{HI}$ efforts in the same players. While no significant differences were

11 obtained, results showed that these final HI actions leading to injury were almost double

12 the length and duration of usual actions. These differences in action length and duration

13 were accompanied by intermediate effect sizes $(>0.5)$. In contrast, the recovery time

14 between the penultimate $\mathrm{HI}$ effort and the HI action leading to injury was shown to be

15 significantly shorter compared to the habitual recovery time between HI bouts

$16(\mathrm{p}=0.003)$. This difference was accompanied by a large effect size $(>0.8)$.

Insert Table 2 about here.

20 Discussion

21 The aim of this first study was to analyse the physical performance of elite soccer 22 players in competition prior to and during actions leading to injury using motion analyses. Within the limitations of the small sample, the running actions in the injury

24 cases indicated that at the time of injury, players were generally moving at moderate

25 speeds after having started their final run at low speeds before attaining high intensities 
1 and then reducing speed. Analysis of the characteristics of high-intensity bouts in the

2 injury situation showed that these actions were greater in both distance and duration,

3 albeit non-significantly, compared to the player's usual efforts. While the players'

4 overall efforts in the 5-minute period leading up to injury did not seem to play a direct

5 part in the injury, a significantly lower than normal recovery time between high-

6 intensity actions prior to sustaining injury was observed.

7 A large proportion of injuries sustained in professional soccer players are

8 sustained during running actions [24] but little is known about the characteristics of

9 these movements. In the present study, analysis of the running actions in the match

10 injury situation showed these involved relatively high movement speeds $(\sim 17 \mathrm{~km} / \mathrm{h})$.

11 Review of the injury situations showed that games skills, physical contact and injury

12 avoidance behaviours were also superimposed on the locomotor activities leading to

13 injury. The majority of the final running actions initially involved an acceleration phase

14 from low to high running intensities, attaining speeds of over $23 \mathrm{~km} / \mathrm{h}$ (for example to

15 create space or close a player down), before the player decelerated to moderate speeds

16 (generally when attempting to gain possession) at which the injury occurred. These

17 acceleration and deceleration characteristics of running actions are highly common to

18 the game of soccer. In the latter, impairments in the eccentric muscle contractions

19 involved in such phases may be linked to an increased risk of joint and muscle injury

20 [21]. Indeed, a recent review on soccer performance has identified a need for specific

21 deceleration exercises in strength and conditioning training sessions [9] which could

22 have some relevance for preventing some of the injuries identified in this and other 23 reports $[24,29,40]$ on soccer match-play.

24 In elite soccer match-play, high-intensity actions are rarely more than 20 metres

25 in length and greater than 4 seconds in duration $[14,18]$. The present results from the 
1 normative analysis of player performance confirm this trend. While no significant

2 differences were reported in distance or duration for the high-intensity run that led to

3 injury, values for these measures were almost twice that of the normative profile. This

4 finding suggests that players may be more at risk of injury when subjected to high-

5 intensity bouts of exercise that are greater than usual in intensity. This observation

6 could have important implications for the design of high-intensity running regimens.

7 However, in the majority of the high-intensity actions that led to injury, players were in

8 situations where they were challenging for possession. Further work combining injury

9 and physical performance data is needed to examine whether the risk of injury is greater

10 in situations where the player is tackling or being tackled which simply happen to be

11 accompanied by high-intensity activities.

12 The physical efforts in the five minutes prior to the injury situation did not seem

13 to play a role in causing injury. While players covered a slightly higher overall distance

$14(+4 \%)$ than they would during a typical five-minute period of play, this result was not

15 significant implying that fatigue was not a contributing factor to injury. However, the

16 players had covered $35 \%$ more distance at high-intensities than usual before sustaining

17 injury. Previous research in soccer match-play has shown that sprint performance is

18 significantly reduced after the most intense periods of exercise indicating that players

19 experience temporary fatigue $[9,28,30]$. The present players may have been

20 experiencing some degree of fatigue due to their increased efforts notably at high-

21 intensities thereby affecting their capacity to perform maximally during the injury

22 action.

23 These findings are supported by a significantly shorter than usual recovery time

24 ( $\sim 36$ seconds versus $\sim 99$ seconds in typical performance) observed between the

25 penultimate high-intensity action and the one that led to injury. During multiple sprint 
1 work, fatigue is manifested as a progressive decline in functional performance, the

2 magnitude of which is largely determined by the duration of the intervening recovery

3 periods [18]. Therefore, at the time of injury, the present players may have experienced

4 transient fatigue due to incomplete recovery between high-intensity bouts increasing

5 their susceptibility to injury. This reduction could have affected functional performance

6 in areas such as proprioceptive ability [37], dynamic joint stability [25], force

7 production [4], neuromuscular responses [19] or running mechanics [38]. However,

8 caution is required when interpreting these results as to the validity of the method

9 employed for determining a normative profile for each player over a 5-minute period.

10 The work-rate pattern in soccer is random, can vary greatly across match periods and

11 between games and depends on many factors such as scoreline or opposition standard.

12 Nevertheless, researchers should be encouraged on the basis of the present study design

13 and results to explore other means for creating a normative profile for work rate over

14 pre-defined match periods.

15 These results may have important implications for the design of conditioning 16 regimes to improve player fitness in an attempt to reduce the risk of injury. For 17 example, high-intensity fitness training in soccer traditionally aims to improve the 18 player's ability to recover quickly following successive bouts of high-intensity 19 anaerobic efforts. This effect is achieved through an increased aerobic response, 20 improved lactate removal, and enhanced phosphocreatine regeneration [18]. Specific 21 high-intensity training regimens are traditionally based on work-rest ratios involving the 22 repetition of runs over a set distance and with fixed recovery times [15]. However, the 23 intensity of actions and recovery periods can alternate at any time according to the 24 demands of the match which was the case during the injury incidents reported in this 25 study. Therefore, to optimise player fitness and reduce the chance of injury, 
1 practitioners could consider constructing high-intensity training programmes on the

2 repetition of runs that vary in both intensity (duration, distance) and in recovery time. These preliminary findings may also be pertinent for constructing exercise

4 protocols to simulate the exercise intensities experienced during match-play to 5 investigate the effects of fatigue on injury risk in muscle and joints. Previous 6 experiments have successfully used soccer-specific exercise within the laboratory 7 setting [20,21,31,33,34,39] to monitor changes in performance. However, these 8 protocols tend to measure fatigue after fixed durations (e.g., start, middle and end of 9 exercise) and use a 90-minute exercise period divided into 15-minute normative activity 10 profiles that are identical in terms of intensity. These exercise protocols therefore do not 11 represent the random nature of activity in soccer and on the basis of the present 12 findings, we suggest that future research using such protocols should also investigate 13 impairments in performance after periods of higher than average exercise intensity.

14 A major limitation of this study was the small number of injury cases examined.

15 In studies of risk factors for sports injuries, a minimum of 20-50 injury cases is 16 recommended [6]. It is inevitable though through comparisons with the low sample 17 sizes obtained in other studies $[2,4]$, that identifying large numbers of real time-loss 18 injuries on video during elite soccer competition is difficult. This limitation of the low 19 sample size is partly countered by the strict injury inclusion criteria combined with the 20 simultaneous access to medical information from team medical staff resulting in a less 21 biased description of how soccer injuries occur [3]. A further limitation was the cohort 22 included players from only one soccer club (as detailed medical information on opposition injuries and physical performance was not available) and the patterns 24 observed may only be a reflection of this particular team. Furthermore, the proportion of 25 injury types (for example, $60 \%$ of injuries were sprains) investigated may not fully 
1 represent the patterns of injury habitually reported in elite soccer match-play $[24,40]$.

2 Investigations involving a substantially larger sample of clubs and injury cases are

3 therefore warranted. However, obtaining confidential information and in sufficient

4 quantities on both physical performance and player injury during match-play from elite

5 clubs is difficult. Similarly, no single research approach to identifying the reasons for

6 injury is adequate in terms of completeness of information provided and it is necessary

7 to combine a number of different approaches to describe the mechanisms fully [27]. For

8 example, the impact of extrinsic factors such as the playing situation, game context and

9 opponent behaviour could have affected the present findings. Nevertheless, important

10 practical insights can be gained from studying the events preceding injury and this first

11 investigation is a first step in identifying and understanding the relationship between

12 physical performance and injury in elite soccer competition. It is hoped that similar

13 research on injury data will be done in other professional clubs and associations to build

14 upon the present findings by exploring some of the gaps and questions identified in this

15 report.

\section{Conclusions}

18 The physical demands of contemporary professional soccer match-play are high and

19 players are subjected to fatigue and risk of injury. The present study is the first to have

20 shown that information on physical performance obtained from motion analyses of

21 match-play may be valuable in increasing knowledge about the events involved in the

22 occurrence of injury. These preliminary findings suggest that when there is inadequate

23 time for recovery between high-intensity exercise bouts and the distance and duration of

24 these actions are substantially higher than usual, players may be at increased risk of

25 sustaining injury. Also, analysis of injuries showed that running actions involved both 
1 an acceleration phase to achieve high speeds as well as a deceleration phase during

2 which injuries were sustained. Whilst further research is evidently necessary, the

3 findings from this and future studies may eventually be employed in injury prevention

$4 \quad$ strategies by informing the prescription of specific fitness training protocols. 


\section{$1 \quad$ References}

21 Andersen TE, Árnason Á, Engebretsen, L, R Bahr. Mechanisms of head injuries in

3 elite football. Br J Sports Med 2004; 38: 690-696

42 Andersen TE, Larsen $\varnothing$, Tenga A, Engebretsen L, Bahr R. Football incident analysis:

5 a new video based method to describe injury mechanisms in professional football.

$6 \quad$ Br J Sports Med 2003; 37: 226-232

73 Andersen TE, Tenga A, Engebretsen L, Bahr R. Video analysis of injuries and 8 incidents in Norwegian professional football. Br J Sports Med 2004; 38: 626-631

94 Apriantono T, Hiroyuki N, Yasuo I, Shinya S. The effect of muscle fatigue on instep 10 kicking kinetics and kinematics in association football. J Sports Sci 2006; 24: 9511960

125 Arnason A, Tenga A, Engebretsen L, Bahr R. A prospective video-based analysis of 13 injury situations in elite male football. Am J Sports Med 2004; 32: 1459-1465

146 Bahr R, Holme I. Risk factors for sports injuries-a methodological approach. Br J $15 \quad$ Sports Med 2003: $37 ; 384-92$

167 Bahr R, Krosshaug T. Understanding injury mechanisms: a key component of 17 preventing injuries in sport. Br J Sports Med 2005; 39; 324-329

188 Bradley PS, Sheldon W, Wooster B, Olsen P, Boanas P, Krustrup P. High-intensity 19 running in English FA Premier League soccer matches. J Sports Sci 2009; 15: 159$20 \quad 68$

219 Carling C, Bloomfield J, Nelsen L, Reilly T. The role of motion analysis in elite 22 soccer: Contemporary performance measurement techniques and work-rate data. $23 \quad$ Sports Med 2008; 38: 839-862

2410 Carling C, Williams AM, Reilly T. The Handbook of Soccer Match Analysis. 25 London: Routledge; 2005 
111 Carling C, Bloomfield J. The effect of an early dismissal on player work-rate in a

2 professional soccer match. J Sci Med in Sport. doi:10.1016/j.jsams.2008.09.004

312 Carling C, Espié V, Le Gall F, Bloomfield J, Reilly T. Work-rate of substitutes in 4 elite soccer: a preliminary study. J Sci Med in Sport. $5 \quad$ doi:10.1016/j.jsams.2009.02.012

613 Cohen, J. (1988). Statistical power analysis for the behavioural sciences. 2nd ed.

$7 \quad$ Hillsdale, NJ: Lawrence Erlbaum

814 Di Salvo V, Baron R, Tschan H, Calderon Montero FJ, Bachl N, Pigozzi F. 9 Performance characteristics according to playing position in elite soccer. Int J Sports $10 \quad$ Med 2007; 28: 222-227

1115 Dupont G, Akakpo K, Berthoin, S. The effect of in-season, high-intensity interval 12 training in soccer players. J Strength Cond Res 2004; 18: 584-589

1316 Fuller CW, Ekstrand J, Junge A, et al. Consensus statement on injury definitions and 14 data collection procedures in studies of football (soccer) injuries. Br J Sports Med $15 \quad 2006 ; 40: 193-201$

1617 Fuller CW, Smith GL, Junge A, Dvorak J. An Assessment of Player Error as an 17 Injury Causation Factor in International Football. Am J Sports Med 2004; 32: 28S

18 Glaister M. Multiple Sprint Work Physiological Responses, Mechanisms of Fatigue 19 and the Influence of Aerobic Fitness. Sports Med 2005; 35: 757-777

19 Gleeson NP, Reilly T, Mercer TH, Rakowski S, Rees D Influence of acute 21 endurance activity on leg neuromuscular and musculoskeletal performance. Med Sci Sports Exerc 1998; 30: 596-608

20 Greig M. The influence of soccer-specific fatigue on peak isokinetic torque 24 production of the knee flexors and extensors. Am J Sports Med 2008; 36: 14031409 
121 Greig M., Siegler, JC. Soccer-specific fatigue and eccentric hamstrings muscle

$2 \quad$ strength. J Athl Train 2009; 44: 180-184

322 Hagglund M, Walden M, Bahr R, Ekstrand J. Methods for epidemiological study of 4 injuries to professional football players: developing the UEFA model. Br J Sports $5 \quad$ Med. 2005;39:340-346.

623 Hawkins RD, Fuller CW. Risk assessment in professional football: an examination 7 of accidents and incidents in the 1994 World Cup finals. Br J Sports Med $8 \quad 1996 ; 30: 165-70$.

924 Hawkins RD, Hulse MA, Wilkinson C, Hodson A, Gibson M. The association 10 football medical research programme: an audit of injuries in professional football $\mathrm{Br}$ $11 \quad$ J Sports Med 2001; 35: 43-47

1225 Jackson ND, Gutierrez GM, Kaminski T. The effect of fatigue and habituation on 13 the stretch reflex of the ankle musculature. J Electromyography and Kinesiology $14 \quad 2009 ; 19: 75-84$

1526 Junge A, Dvorak J. Soccer injuries: a review on incidence and prevention. Sports $16 \quad$ Med 2004; 34: 929-38

1727 Krosshaug T, Andersen TE, Olsen O-E, Myklebust G, Bahr R. Research approaches 18 to describe the mechanisms of injuries in sport: limitations and possibilities. $\mathrm{Br} \mathrm{J}$ Sports Med 2005; 39: 330-339

28 Krustrup P, Mohr M, Steensberg A, Bencke J, Kjaer M, Bangsbo J. Muscle and blood metabolites during a soccer game: implications for sprint performance. Med Sci Sports Exerc 2006: 38; 1165-1174

29 Le Gall F, Carling C, Reilly T, Church, J, Rochcongar P. Incidence of injuries in 24 elite French youth soccer players: a 10-season study. Am J Sports Med 2006; 34: 928-938 
130 Mohr M, Krustrup P, Bangsbo J. Match performance of high-standard soccer

2 players with special reference to development of fatigue. J Sports Sci. 2003; 21:

$3 \quad 519-528$

431 Oliver J, Armstrong N, Williams C. Changes in jump performance and muscle 5 activity following soccer-specific exercise. J Sports Sci 2007; 26: 141-148

632 Rahnama N, Reilly T, Lees A. Injury risk associated with playing actions during $7 \quad$ competitive soccer. Br J Sports Med 2002; 36: 354-359

833 Rahnama N, Reilly T, Lees A. Electromyography of selected lower-limb muscles

9 fatigued by exercise at the intensity of soccer match-play. J Electromyography and

$10 \quad$ Kinesiology 2006; 16: 257-263

1134 Rahnama N, Reilly T, Lees A, Graham-Smith P. Muscle fatigue induced by exercise 12 simulating the work rate of competitive soccer. J Sports Sci 2003; 21: 933-942

1335 Rampinini E, Coutts AJ, Castagna C, Sassi R, Impellizzeri FM. Variation in top 14 level soccer match performance. Int J Sports Med 2007; 28: 1018-1024

1536 Rampinini, E Impellizzeri FM, Castagna, C, Coutts A, Wisløff U. Technical 16 performance during soccer matches of the Italian Serie A league: Effect of fatigue 17 and competitive level. J Sci Med Sport 2009; 12: 227-233

1837 Rozzi SL, Lephart SM, Fu FH. Effects of muscular fatigue on knee joint laxity and 19 neuromuscular characteristics of male and female athletes. J. Athl. Train 1999; 34: $106-114$

2138 Sanna G, O’Connor K. Fatigue-related changes in stance leg mechanics during 22 sidestep cutting maneuvers. Clin Biomech 2008; 23: 946-954

39 Small K, McNaughton L, Greig M, Lovell R. The effects of multidirectional soccer24 specific fatigue on markers of hamstring injury risk. J Sci Med Sport, (in press). 
140 Waldén M, Hägglund M, Ekstrand J. UEFA Champions League study: a prospective

2 study of injuries in professional football during the 2001-2002 season. Br J Sports

$3 \quad$ Med 2005;39(8):542-6

441 Zubillaga A. La actividad del jugador de fútbol en alta competición: análisis de

$5 \quad$ variabilidad [PhD Thesis]. Spain; Malaga: University of Sport Sciences and

$6 \quad$ Exercise, 2006 
42 Table 1 Descriptive characteristics of injuries and movement data for the final running action leading to the injury.

2

\begin{tabular}{|c|c|c|c|c|c|c|c|c|c|c|c|c|c|}
\hline \multirow[b]{2}{*}{ Position } & \multirow[b]{2}{*}{ Type } & \multicolumn{2}{|l|}{ Injury } & \multirow[t]{2}{*}{ Layoff time } & \multicolumn{4}{|c|}{ Speed $(\mathrm{km} / \mathrm{h})$} & \multirow{2}{*}{$\begin{array}{l}\text { Duration } \\
\text { (s) }\end{array}$} & \multirow{2}{*}{$\begin{array}{l}\text { Length } \\
\text { (m) }\end{array}$} & \multirow{2}{*}{$\begin{array}{l}\text { In ball } \\
\text { Possession }\end{array}$} & \multirow[t]{2}{*}{ Contact } & \multirow[t]{2}{*}{ Qualitative description of game incident } \\
\hline & & Location & Time in match & & Start & At injury & Maximal & Average & & & & & \\
\hline Defender & Sprain & Ankle & 73rd minute & $>1$ month & 0.6 & 8,0 & 12.1 & 9,0 & 1.6 & 4,0 & No & No & Run then jump to head ball and injured when taking off \\
\hline Defender & Sprain & Ankle & 36th minute & 1-4 weeks & 12.7 & 17.5 & 19.2 & 18,0 & 2,0 & 10,0 & No & Yes & $\begin{array}{l}\text { Run to intercept ball and injured during challenge for possession } \\
\text { Run to close down opposition player followed by turn and injured during }\end{array}$ \\
\hline Defender & Sprain & Knee & 7th minute & $1-4$ weeks & 8,0 & 16.4 & 23.7 & 18.6 & 8.8 & 45.5 & No & No & ensuing run \\
\hline Defender & Sprain & Knee & 85th minute & $1-4$ weeks & 5.3 & 11.7 & 21.2 & 18,0 & 2,0 & 10,0 & No & Yes & Run to intercept ball and injured during landing after challenge for possession \\
\hline Defender & Sprain & Ankle & 7th minute & $1-4$ weeks & 10.9 & 11,0 & 11.3 & 11,0 & 2.5 & 11,0 & No & Yes & Run to cover space and injured while challenging for possession \\
\hline $\begin{array}{l}\text { Defender } \\
\text { Centre- }\end{array}$ & Strain & Groin & 32nd minute & 1-4 weeks & 1.8 & 16.9 & 30,0 & 20.9 & 11.4 & 66.4 & No & No & $\begin{array}{l}\text { Run to meet pass and injured when controlling ball } \\
\text { Run to close down opposition plaver followed by turn and injured during }\end{array}$ \\
\hline $\begin{array}{l}\text { forward } \\
\text { Centre- }\end{array}$ & Fracture/dislocation & Ankle/Lower leg & 25th minute & $>1$ month & 5,0 & 12.8 & 22,0 & 16.1 & 5.6 & 25.1 & No & Yes & ensuing tackle \\
\hline $\begin{array}{l}\text { forward } \\
\text { Centre- }\end{array}$ & Bruising/Hematoma & Thigh & 24th minute & 1-4 weeks & 5.4 & 9.4 & 26.6 & 21.2 & 11,0 & 64.7 & No & Yes & Run to meet pass and injured during challenge for possession \\
\hline $\begin{array}{l}\text { forward } \\
\text { Centre- }\end{array}$ & Sprain & Ankle & 16th minute & $1-4$ weeks & 9.2 & 19.3 & 30.2 & 16.9 & 5.1 & 24,0 & No & Yes & Run into space and injured during challenge for possession \\
\hline forward & Strain & Hamstring & 23rd minute & 1-4 weeks & 2.9 & 18.7 & 22.4 & 16.2 & 6.5 & 29.2 & Yes & No & Run into space to collect possession and injured when passing \\
\hline Mean \pm SD & & & & & $6.2 \pm 4.0$ & $14.2 \pm 4.2$ & $23.0 \pm 6.5$ & $16.6 \pm 3.8$ & $5.7 \pm 3.7$ & $29.0 \pm 22.7$ & & & \\
\hline
\end{tabular}


47

2 Table 2 Comparison between the players' normative physical performance profile over a typical 5-minute game period and the 5-minute period

3 leading up to the injury; and comparison between the normative physical performance profile for a typical high-intensity action and the high-

4 intensity action during which injury occurred.

5

48

\begin{tabular}{lllrl}
\hline & Normative & Injury Action & Mean difference & Effect Size \\
& & & & \\
\hline $\begin{array}{l}\text { Distance covered in 5-min period } \\
\text { leading to injury (m) }\end{array}$ & & & & \\
$\quad$ Total & $569.2 \pm 28.2$ & $594.5 \pm 88.3$ & $25.3 \pm 84.8$ & 0.36 \\
$\quad$ High-intensity & $44.2 \pm 11.2$ & $67.0 \pm 57.3$ & $22.8 \pm 61.9$ & 0.53 \\
$\quad$ Moderate-intensity & $82.0 \pm 14.0$ & $84.8 \pm 45.5$ & $2.8 \pm 47.3$ & 0.06 \\
$\quad$ Low-intensity & $81.7 \pm 15.2$ & $87,1 \pm 31.4$ & $5.4 \pm 30.6$ & 0.19 \\
$\quad$ Walking/jogging & $361.4 \pm 6.0$ & $355.6 \pm 59.9$ & $-5.8 \pm 59.9$ & 0.08 \\
& & & & \\
Characteristics of final high-intensity & & & & \\
runs leading to injury (n=8) & $15.7 \pm 0.4$ & $27.6 \pm 18.7$ & $10.9 \pm 18.8$ & 0.71 \\
$\quad$ Length (m) & $2.5 \pm 0.1$ & $4.2 \pm 2.7$ & $1.7 \pm 2.7$ & 0.50 \\
$\quad \begin{array}{l}\text { Duration (s) } \\
\text { Speed (km/h) }\end{array}$ & $22.2 \pm 0.2$ & $22.0 \pm 1.7$ & $-0.2 \pm 1.8$ & 0.00 \\
$\quad \begin{array}{l}\text { Recovery time after previous } \\
\text { high-intensity run (s) }\end{array}$ & $98.8 \pm 17.5 *$ & $35.6 \pm 16.8$ & $-63.2 \pm 26.6$ & 3.50 \\
\hline
\end{tabular}

$6 \quad 49$

$750 *$ Significantly greater recovery time $(\mathrm{p}=0.003)$

51

9 52Mean \pm SD

$10 \quad 53$ 